\title{
Hypervector Spaces Based on Intersectional Soft Sets
}

\author{
Young Bae Jun, ${ }^{1}$ Chul Hwan Park, ${ }^{2}$ and N. O. Alshehri ${ }^{3}$ \\ ${ }^{1}$ Department of Mathematics Education (and RINS), Gyeongsang National University, Jinju 660-701, Republic of Korea \\ ${ }^{2}$ School of Digital Mechanics, Ulsan College, Nam-Gu Ulsan 680-749, Republic of Korea \\ ${ }^{3}$ Department of Mathematics, Faculty of Science for Girls, King Abdulaziz University, Jeddah, Saudi Arabia
}

Correspondence should be addressed to N. O. Alshehri; nalshehrie@kau.edu.sa

Received 25 November 2013; Accepted 7 January 2014; Published 4 March 2014

Academic Editor: Gerd Teschke

Copyright (C) 2014 Young Bae Jun et al. This is an open access article distributed under the Creative Commons Attribution License, which permits unrestricted use, distribution, and reproduction in any medium, provided the original work is properly cited.

The notion of int-soft subfields, int-soft algebras over int-soft subfields, and int-soft hypervector spaces are introduced, and their properties and characterizations are considered. In connection with linear transformations, int-soft hypervector spaces are discussed.

\section{Introduction}

The hyperstructure theory was introduced by Marty [1] at the 8th congress of Scandinavian Mathematicians in 1934. Since then many researchers have worked in these areas, for example, hyper-MV-algebras, hyper-BCK/BCI/BCCalgebras, hyper- $B L$-algebras, hyperlattices, and so forth. As a generalization of fuzzy vector spaces, the fuzzy hypervector spaces are studied by Ameri and Dehghan (see [2,3]).

Various problems in system identification involve characteristics which are essentially nonprobabilistic in nature [4]. In response to this situation Zadeh [5] introduced fuzzy set theory as an alternative to probability theory. Uncertainty is an attribute of information. In order to suggest a more general framework, the approach to uncertainty is outlined by Zadeh [6]. To solve complicated problem in economics, engineering, and environment, we cannot successfully use classical methods because of various uncertainties typical for those problems. There are three theories: theory of probability, theory of fuzzy sets, and the interval mathematics which we can consider as mathematical tools for dealing with uncertainties. But all these theories have their own difficulties. Uncertainties cannot be handled using traditional mathematical tools but may be dealt with using a wide range of existing theories such as probability theory, theory of (intuitionistic) fuzzy sets, theory of vague sets, theory of interval mathematics, and theory of rough sets. However, all of these theories have their own difficulties which are pointed out in [7]. Maji et al. [8] and Molodtsov [7] suggested that one reason for these difficulties may be due to the inadequacy of the parametrization tool of the theory. To overcome these difficulties, Molodtsov [7] introduced the concept of soft set as a new mathematical tool for dealing with uncertainties that is free from the difficulties that have troubled the usual theoretical approaches. Molodtsov pointed out several directions for the applications of soft sets. At present, works on the soft set theory are progressing rapidly. Maji et al. [8] described the application of soft set theory to a decision making problem. Maji et al. [9] also studied several operations on the theory of soft sets. Chen et al. [10] presented a new definition of soft set parametrization reduction and compared this definition to the related concept of attributes reduction in rough set theory. The algebraic structure of set theories dealing with uncertainties has been studied by some authors. Çağman et al. [11] introduced fuzzy parameterized (FP) soft sets and their related properties. They proposed a decision making method based on FP-soft set theory and provided an example which shows that the method can be successfully applied to the problems that contain uncertainties. Feng [12] considered the application of soft rough approximations in multicriteria group decision making problems. Aktaş and Çağman [13] studied the basic concepts of soft set theory and compared soft sets to fuzzy and rough sets, providing examples to clarify their differences. 
They also discussed the notion of soft groups. After that, many algebraic properties of soft sets are studied (see [1424]).

In this paper, we introduce the notion of int-soft subfields, int-soft algebras over int-soft subfields, and int-soft hypervector spaces. We study their properties and characterizations. In connection with linear transformations, we discuss int-soft hypervector spaces.

\section{Preliminaries}

A soft set theory is introduced by Molodtsov [7], and Çağman and Enginoğlu [25] provided new definitions and various results on soft set theory.

In what follows, let $U$ be an initial universe set and $E$ be a set of parameters. Let $\mathscr{P}(U)$ denote the power set of $U$ and $A, B, C, \ldots \subseteq E$.

Definition 1 (see $[7,25])$. A soft set $(\tilde{f}, A)$ over $U$ is defined to be the set of ordered pairs

$$
(\tilde{f}, A):=\{(x, \tilde{f}(x)): x \in E, \tilde{f}(x) \in \mathscr{P}(U)\},
$$

where $\tilde{f}: E \rightarrow \mathscr{P}(U)$ such that $\tilde{f}(x)=\emptyset$ if $x \notin A$.

For any sets $X$ and $Y$, let $\mu: X \rightarrow Y$ be a function and $(\tilde{f}, X)$ and $(\tilde{g}, Y)$ be soft sets over $U$.

(1) The soft set

$\mu^{-1}(\widetilde{g}, Y)=\left\{\left(x, \mu^{-1}(\widetilde{g})(x)\right): x \in X, \mu^{-1}(\widetilde{g})(x) \in \mathscr{P}(U)\right\}$,

where $\mu^{-1}(\widetilde{g})(x)=\widetilde{g}(\mu(x))$, is called the soft preimage of $(\tilde{g}, Y)$ under $\mu$.

(2) The soft set

$$
\mu(\widetilde{f}, X)=\{(y, \mu(\widetilde{f})(y)): y \in Y, \mu(\widetilde{f})(y) \in \mathscr{P}(U)\},
$$

where

$$
\mu(\tilde{f})(y)= \begin{cases}\bigcup_{x \in \mu^{-1}(y)} \tilde{f}(x) & \text { if } \mu^{-1}(y) \neq \emptyset \\ \emptyset & \text { otherwise }\end{cases}
$$

is called the soft image of $(\tilde{f}, X)$ under $\mu$.

A map $\circ: H \times H \rightarrow P_{*}(H)$ is called a hyperoperation or join operation, where $P_{*}(H)$ is the set of all nonempty subsets of $H$. The join operation is extended to subsets of $H$ in natural way, so that $A \circ B$ is given by

$$
A \circ B=\bigcup\{a \circ b \mid a \in A, b \in B\} .
$$

The notations $a \circ A$ and $A \circ a$ are used for $\{a\} \circ A$ and $A \circ$ $\{a\}$, respectively. Generally, the singleton $\{a\}$ is identified by its element $a$.
Definition 2 (see [26]). Let $F$ be a field and $(V,+)$ be an abelian group. A hypervector space over $F$ is defined to be the quadruplet $(V,+, \circ, F)$, where "o" is a mapping

$$
\circ: F \times V \longrightarrow P_{*}(V)
$$

such that for all $a, b \in F$ and $x, y \in V$ the following conditions hold:

$$
\begin{aligned}
& \text { (H1) } a \circ(x+y) \subseteq a \circ x+a \circ y, \\
& (\mathrm{H} 2)(a+b) \circ x \subseteq a \circ x+b \circ x, \\
& (\mathrm{H} 3) a \circ(b \circ x)=(a b) \circ x, \\
& (\mathrm{H} 4) a \circ(-x)=(-a) \circ x=-(a \circ x), \\
& (\mathrm{H} 5) x \in 1 \circ x .
\end{aligned}
$$

A hypervector space $(V,+, \circ, F)$ over a field $F$ is said to be strongly left distributive (see [2]) if it satisfies the following condition:

$$
(\forall a \in F) \quad(\forall x, y \in V) \quad(a \circ(x+y)=a \circ x+a \circ y) .
$$

\section{Int-Soft Algebras over an Int-Soft Field}

In what follows let $F$ be a field unless otherwise specified.

Definition 3. A soft set $(\tilde{f}, F)$ over $F$ is called an int-soft subfield of $F$ if the following conditions are satisfied:

(1) $(\forall a, b \in F)(\tilde{f}(a+b) \supseteq \widetilde{f}(a) \cap \tilde{f}(b))$,

(2) $(\forall a \in F)(\tilde{f}(-a) \supseteq \tilde{f}(a))$,

(3) $(\forall a, b \in F)(\widetilde{f}(a) \cap \tilde{f}(b) \subseteq \widetilde{f}(a b))$,

(4) $(\forall a \in F)\left(a \neq 0 \Rightarrow \tilde{f}(a) \subseteq \tilde{f}\left(a^{-1}\right)\right)$.

Proposition 4. If $(\tilde{f}, F)$ is an int-soft subfield of $F$, then

(1) $(\forall a \in F)(\tilde{f}(a) \subseteq \tilde{f}(0))$,

(2) $(\forall a \in F)(a \neq 0 \Rightarrow \widetilde{f}(a) \subseteq \widetilde{f}(1))$,

(3) $\tilde{f}(1) \subseteq \tilde{f}(0)$.

Proof. (1) For all $a \in F$, we have

$$
\tilde{f}(0)=\tilde{f}(a+(-a)) \supseteq \tilde{f}(a) \cap \tilde{f}(-a)=\tilde{f}(a) .
$$

(2) Let $a \in F$ be such that $a \neq 0$. Then

$$
\tilde{f}(1)=\tilde{f}\left(a a^{-1}\right) \supseteq \tilde{f}(a) \cap \tilde{f}\left(a^{-1}\right)=\tilde{f}(a) .
$$

(3) It is by (1).

It is easy to show that the following theorem holds.

Theorem 5. A soft set $(\tilde{f}, F)$ over $F$ is an int-soft subfield of $F$ if and only if the nonempty $\gamma$-inclusive set

$$
i_{F}(\tilde{f} ; \gamma):=\{a \in F \mid \gamma \subseteq \tilde{f}(a)\}
$$

of $(\tilde{f}, F)$ is a subfield of $F$ for all $\gamma \in \mathscr{P}(U)$. 
Definition 6. Let $V$ be an algebra over $F$ and let $(\tilde{f}, F)$ be an int-soft subfield of $F$. A soft set $(\widetilde{g}, V)$ is called an int-soft algebra over $(\tilde{f}, F)$ if it satisfies the following conditions:

(1) $(\forall x, y \in V)(\widetilde{g}(x+y) \supseteq \widetilde{g}(x) \cap \widetilde{g}(y))$,

(2) $(\forall a \in F)(\forall x \in V)(\widetilde{g}(a x) \supseteq \widetilde{f}(a) \cap \tilde{g}(x))$,

(3) $(\forall x, y \in V)(\widetilde{g}(x y) \supseteq \widetilde{g}(x) \cap \tilde{g}(y))$,

(4) $(\forall x \in V)(\tilde{f}(1) \supseteq \widetilde{g}(x))$.

Proposition 7. Let $V$ be an algebra over $F$ and let $(\tilde{f}, F)$ be an int-soft subfield of $F$. If $(\widetilde{g}, V)$ is an int-soft algebra over $(\tilde{f}, F)$, then $\widetilde{f}(0) \supseteq \widetilde{g}(x)$ for all $x \in V$.

Proof. For any $x \in V$, we have $\widetilde{f}(0) \supseteq \widetilde{f}(1) \supseteq \widetilde{g}(x)$.

Theorem 8. Let $V$ be an algebra over $F$ and let $(\tilde{f}, F)$ be an int-soft subfield of $F$. Then a soft set $(\widetilde{g}, V)$ is an int-soft algebra $\operatorname{over}(\tilde{f}, F)$ if and only if the following conditions are valid:

(1) $(\forall a, b \in F)(\forall x, y \in V)(\widetilde{g}(a x+b y) \supseteq(\tilde{f}(a) \cap \widetilde{g}(x)) \cap$ $(\widetilde{f}(b) \cap \widetilde{g}(y)))$,

(2) $(\forall x, y \in V)(\widetilde{g}(x y) \supseteq \widetilde{g}(x) \cap \widetilde{g}(y))$,

(3) $(\forall x \in V)(\widetilde{f}(1) \supseteq \widetilde{g}(x))$.

Proof. Assume that $(\widetilde{g}, V)$ is an algebra over $(\tilde{f}, F)$. Using (1) and (2) of Definition 6, we have

$$
\begin{aligned}
\widetilde{g}(a x+b y) & \supseteq \widetilde{g}(a x) \cap \widetilde{g}(b y) \\
& \supseteq(\tilde{f}(a) \cap \widetilde{g}(x)) \cap(\tilde{f}(b) \cap \widetilde{g}(y))
\end{aligned}
$$

for all $a, b \in F$ and $x, y \in V$.

Conditions (2) and (3) are by Definition 6 (3) and Definition 6 (4), respectively.

Conversely, suppose that the inclusions of Theorem 8 hold for all $a, b \in F$ and $x, y \in V$. Then

$$
\begin{aligned}
\tilde{g}(x+y) & =\widetilde{g}(1 x+1 y) \supseteq(\tilde{f}(1) \cap \tilde{g}(x)) \cap(\tilde{f}(1) \cap \tilde{g}(y)) \\
& =\widetilde{g}(x) \cap \tilde{g}(y) .
\end{aligned}
$$

The condition (3) and Proposition 4 (3) imply that $\widetilde{f}(0) \supseteq$ $\tilde{f}(1) \supseteq \widetilde{g}(x)$ for all $x \in V$. Thus

$$
\begin{aligned}
\widetilde{g}(a x) & =\widetilde{g}(a x+0 x) \supseteq(\widetilde{f}(a) \cap \widetilde{g}(x)) \cap(\widetilde{f}(0) \cap \widetilde{g}(x)) \\
& =(\widetilde{f}(a) \cap \tilde{g}(x)) \cap \tilde{g}(x)=\tilde{f}(a) \cap \widetilde{g}(x)
\end{aligned}
$$

for all $a \in F$ and $x \in V$. Therefore $(\widetilde{g}, V)$ is an algebra over $(\tilde{f}, F)$.

Theorem 9. Let $V$ and $W$ be algebras over $F$. For any algebraic homomorphism $\mu: V \rightarrow W$, we have the following.
(1) If $(\widetilde{g}, W)$ is an int-soft algebra over $(\tilde{f}, F)$, then the soft preimage $\mu^{-1}(\widetilde{g}, W)$ of $(\widetilde{g}, W)$ under $\mu$ is also an intsoft algebra over $(\tilde{f}, F)$.

(2) If $(\widetilde{g}, V)$ is an int-soft algebra over $(\tilde{f}, F)$, then the soft image $\mu(\widetilde{g}, V)$ of $(\widetilde{g}, V)$ under $\mu$ is also an int-soft algebra over $(\tilde{f}, F)$.

Proof. (1) For any $a, b \in F$ and $x, y \in V$, we have

$$
\begin{aligned}
\mu^{-1} & (\widetilde{g})(a x+b y) \\
& =\widetilde{g}(\mu(a x+b y))=\tilde{g}(a \mu(x)+b \mu(y)) \\
& \supseteq(\tilde{f}(a) \cap \tilde{g}(\mu(x))) \cap(\tilde{f}(b) \cap \tilde{g}(\mu(y))) \\
& =\left(\tilde{f}(a) \cap \mu^{-1}(\tilde{g})(x)\right) \cap\left(\tilde{f}(b) \cap \mu^{-1}(\tilde{g})(y)\right), \\
\mu^{-1}(\tilde{g})(x y)= & \tilde{g}(\mu(x y))=\tilde{g}(\mu(x) \mu(y)) \\
\supseteq & \tilde{g}(\mu(x)) \cap \tilde{g}(\mu(y)) \\
= & \mu^{-1}(\widetilde{g})(x) \cap \mu^{-1}(\tilde{g})(y),
\end{aligned}
$$

and $\widetilde{f}(1) \supseteq \widetilde{g}(\mu(x))=\mu^{-1}(\widetilde{g})(x)$. Therefore, by Theorem 8 , $\mu^{-1}(\widetilde{g}, W)$ is an algebra over $(\widetilde{f}, F)$.

(2) Let $y_{1}, y_{2} \in W$. If $\mu^{-1}\left(y_{1}\right)=\emptyset$ or $\mu^{-1}\left(y_{2}\right)=\emptyset$, then

$$
\mu(\widetilde{g})\left(y_{1}\right) \cap \mu(\widetilde{g})\left(y_{2}\right)=\emptyset \subseteq \mu(\widetilde{g})\left(y_{1}+y_{2}\right) .
$$

Assume that $\mu^{-1}\left(y_{1}\right) \neq \emptyset$ and $\mu^{-1}\left(y_{2}\right) \neq \emptyset$. Then $\mu^{-1}\left(y_{1}+\right.$ $\left.y_{2}\right) \neq \emptyset$, and so

$$
\begin{aligned}
\mu(\tilde{g})\left(y_{1}+y_{2}\right) & =\bigcup_{x \in \mu^{-1}\left(y_{1}+y_{2}\right)} \tilde{g}(x) \supseteq \bigcup_{\substack{x_{1} \in \mu^{-1}\left(y_{1}\right) \\
x_{2} \in \mu^{-1}\left(y_{2}\right)}} \tilde{g}\left(x_{1}+x_{2}\right) \\
& \supseteq \bigcup_{\substack{x_{1} \in \mu^{-1}\left(y_{1}\right) \\
x_{2} \in \mu^{-1}\left(y_{2}\right)}}\left(\widetilde{g}\left(x_{1}\right) \cap \tilde{g}\left(x_{2}\right)\right) \\
& =\left(\bigcup_{x_{1} \in \mu^{-1}\left(y_{1}\right)} \tilde{g}\left(x_{1}\right)\right) \cap\left(\bigcup_{x_{2} \in \mu^{-1}\left(y_{2}\right)} \tilde{g}\left(x_{2}\right)\right) \\
& =\mu(\tilde{g})\left(y_{1}\right) \cap \mu(\tilde{g})\left(y_{2}\right) .
\end{aligned}
$$

For any $y \in W$ and $a \in F$, we have

$$
\begin{aligned}
\mu(\widetilde{g})(a y) & =\bigcup_{\mu(x)=a y} \tilde{g}(x)=\bigcup_{\mu(x)=y} \tilde{g}(a y) \\
& \supseteq \bigcup_{\mu(x)=y}(\tilde{f}(a) \cap \widetilde{g}(y)) \\
& =\tilde{f}(a) \cap \bigcup_{\mu(x)=y} \tilde{g}(y) \\
& =\tilde{f}(a) \cap \mu(\tilde{g})(y) .
\end{aligned}
$$


For all $y_{1}, y_{2} \in W$, if at least one of $\mu^{-1}\left(y_{1}\right)$ and $\mu^{-1}\left(y_{1}\right)$ is empty, then the inclusion

$$
\mu(\tilde{g})\left(y_{1}\right) \cap \mu(\tilde{g})\left(y_{1}\right) \subseteq \mu(\widetilde{g})\left(y_{1} y_{2}\right)
$$

is clear. Assume that $\mu^{-1}\left(y_{1}\right) \neq \emptyset$ and $\mu^{-1}\left(y_{2}\right) \neq \emptyset$. Then

$$
\begin{aligned}
\mu(\tilde{g}) & \left(y_{1}\right) \cap \mu(\tilde{g})\left(y_{2}\right) \\
= & \left(\bigcup_{x_{1} \in \mu^{-1}\left(y_{1}\right)} \tilde{g}\left(x_{1}\right)\right) \cap\left(\bigcup_{x_{2} \in \mu^{-1}\left(y_{2}\right)} \tilde{g}\left(x_{2}\right)\right) \\
= & \bigcup_{\substack{x_{1} \in \mu^{-1}\left(y_{1}\right) \\
x_{2} \in \mu^{-1}\left(y_{2}\right)}}\left(\tilde{g}\left(x_{1}\right) \cap \tilde{g}\left(x_{2}\right)\right) \\
& \subseteq \bigcup_{\substack{x_{1} \in \mu^{-1}\left(y_{1}\right) \\
x_{2} \in \mu^{-1}\left(y_{2}\right)}} \tilde{g}\left(x_{1} x_{2}\right)=\bigcup_{x \in \mu^{-1}\left(y_{1} y_{2}\right)} \tilde{g}(x) \\
= & \mu(\tilde{g})\left(y_{1} y_{2}\right) .
\end{aligned}
$$

Since $\widetilde{f}(1) \supseteq \widetilde{g}(x)$ for all $x \in V$, it follows that

$$
\tilde{f}(1) \supseteq \bigcup_{x \in \mu^{-1}(y)} \tilde{g}(x)=\mu(\widetilde{g})(y)
$$

for all $y \in W$. Therefore $\mu(\tilde{g}, V)$ is an algebra over $(\tilde{f}, F)$.

\section{Int-Soft Hypervector Spaces}

Definition 10. Let $V$ be a hypervector space over $F$ and $(\tilde{f}, F)$ an int-soft subfield of $F$. A soft set $(\tilde{g}, V)$ over $V$ is called an int-soft hypervector space of $V$ related to $(\widetilde{f}, F)$ if the following assertions are valid:

(1) $(\forall x, y \in V)(\tilde{g}(x+y) \supseteq \tilde{g}(x) \cap \tilde{g}(y))$,

(2) $(\forall x \in V)(\widetilde{g}(-x) \supseteq \widetilde{g}(x))$,

(3) $(\forall a \in F)(\forall x \in V)\left(\bigcap_{y \in a \circ x} \widetilde{g}(y) \supseteq \widetilde{f}(a) \cap \widetilde{g}(x)\right)$,

(4) $\widetilde{f}(1) \supseteq \widetilde{g}(\theta)$ where $\theta$ is the zero of $(V,+)$.

Proposition 11. Let $V$ be a hypervector space over $F$ and $(\tilde{f}, F)$ an int-soft subfield of F. If $(\tilde{g}, V)$ is an int-soft hypervector space of $V$ related to $(\tilde{f}, F)$, then

(1) $\tilde{g}(\theta) \subseteq \tilde{f}(0)$,

(2) $(\forall x \in V)(\widetilde{g}(x) \subseteq \widetilde{g}(\theta))$,

(3) $(\forall x \in V)(\widetilde{g}(x) \subseteq \tilde{f}(0))$.

Proof. It is an immediate consequence of Definition 10 and Proposition 4.

Proposition 12. Let $V$ be a hypervector space over $F$. If $(\tilde{g}, V)$ is an int-soft hypervector space of $V$ related to an int-soft subfield $(\tilde{f}, F)$ of $F$, then

$$
(\forall x \in V)\left(\tilde{g}(x)=\bigcap_{y \in 1 \circ x} \tilde{g}(y)\right) .
$$

Proof. Let $x \in V$. Since $x \in 1 \circ x$ by (H5), we have $\widetilde{g}(x) \supseteq$ $\bigcap_{y \in 1 \circ x} \widetilde{g}(y)$. Using Definition 10 (3)

$$
\bigcap_{y \in 1 \circ x} \tilde{g}(y) \supseteq \tilde{f}(1) \cap \widetilde{g}(x) \supseteq \widetilde{g}(x) .
$$

Hence $\tilde{g}(x)=\bigcap_{y \in 1 \circ x} \tilde{g}(y)$ for all $x \in V$.

Theorem 13. Assume that a hypervector space $V$ over $F$ is strongly left distributive. Let $(\tilde{f}, F)$ be an int-soft subfield of $F$. Then a soft set $(\tilde{g}, V)$ over $V$ is an int-soft hypervector space of $V$ related to $(\tilde{f}, F)$ if and only if the following conditions are true:

(1) $\bigcap_{z \in a \circ x+b \circ y} \tilde{g}(z) \supseteq(\tilde{f}(a) \cap \tilde{g}(x)) \cap(\tilde{f}(b) \cap \tilde{g}(y))$,

(2) $\widetilde{g}(x) \subseteq \widetilde{f}(1)$

for all $a, b \in F$ and all $x, y \in V$.

Proof. Assume that $(\tilde{g}, V)$ is an hypervector space of $V$ related to $(\widetilde{f}, F)$. The second condition follows from Proposition 11 (2) and Definition 10 (4). Let $a, b \in F$ and $x, y \in V$. Then

$$
\begin{aligned}
& \bigcap_{z \in a \circ x+b \circ y} \tilde{g}(z) \\
& =\bigcap_{\substack{z \in u+v \\
u \in a \circ x, v \in b \circ y}} \tilde{g}(z) \supseteq(\tilde{f}(a) \cap \tilde{g}(x)) \cap(\tilde{f}(b) \cap \tilde{g}(y)) .
\end{aligned}
$$

Conversely suppose the conditions (1) and (2) are true. For all $x, y \in V$, we have

$$
\begin{aligned}
\tilde{g}(x+y) & \supseteq \bigcap_{z \in 1 \circ x+1 \circ y} \tilde{g}(z) \\
& \supseteq(\tilde{f}(1) \cap \tilde{g}(x)) \cap(\tilde{f}(1) \cap \tilde{g}(y)) \\
& \supseteq \widetilde{g}(x) \cap \tilde{g}(y) .
\end{aligned}
$$

Since $(\tilde{f}, F)$ is a subfield of $F$, we have $\widetilde{g}(a) \subseteq \widetilde{f}(1) \subseteq \widetilde{f}(0)$ and $\tilde{g}(a) \subseteq \tilde{f}(1) \subseteq \tilde{f}(-1)$. Note that $0 \in 0 \circ x$ for all $x \in V$. It follows that

$$
\begin{aligned}
\tilde{g}(-x) & \supseteq \bigcap_{y \in 0 \circ x+(-1) \circ x} \tilde{g}(y) \\
& \supseteq(\widetilde{f}(0) \cap \widetilde{g}(x)) \cap(\tilde{f}(-1) \cap \tilde{g}(x)) \\
& =\widetilde{g}(x) \cap \tilde{g}(x)=\widetilde{g}(x)
\end{aligned}
$$

for all $x \in V$. Let $a \in F$ and $x \in V$. Then

$$
\begin{aligned}
\bigcap_{y \in a \circ x} \tilde{g}(y) & \supseteq \bigcap_{\substack{y \in u+v \\
u \in 0 \circ x, v \in a \circ x}} \tilde{g}(y) \\
& \supseteq(\tilde{f}(0) \cap \tilde{g}(x)) \cap(\tilde{f}(a) \cap \tilde{g}(x)) \\
& =\tilde{g}(x) \cap(\tilde{f}(a) \cap \tilde{g}(x))=\tilde{f}(a) \cap \tilde{g}(x) .
\end{aligned}
$$

Clearly, $\widetilde{f}(1) \supseteq \widetilde{g}(\theta)$. Therefore $(\tilde{g}, V)$ is an int-soft hypervector space of $V$ related to $(\tilde{f}, F)$. 
Theorem 14. Let $V$ be a hypervector space over $F$ and $(\tilde{f}, F)$ an int-soft subfield of $F$. If a soft set $(\widetilde{g}, V)$ over $V$ is an int-soft hypervector space of $V$ related to $(\tilde{f}, F)$, then the nonempty $\gamma$ inclusive set

$$
i_{V}(\widetilde{g} ; \gamma):=\{x \in V \mid \gamma \subseteq \tilde{g}(x)\}
$$

of $(\tilde{g}, V)$ is a subhypervector space of $V$ over the field $i_{F}(\tilde{f} ; \gamma)$ for all $\gamma \in \mathscr{P}(U)$.

Proof. Let $x, y \in i_{V}(\widetilde{g} ; \gamma)$. Then $\widetilde{g}(x) \supseteq \gamma$ and $\widetilde{g}(y) \supseteq \gamma$. It follows that

$$
\begin{aligned}
\widetilde{g}(x-y) & =\widetilde{g}(x+(-y)) \supseteq \widetilde{g}(x) \cap \widetilde{g}(-y) \\
& \supseteq \widetilde{g}(x) \cap \widetilde{g}(y) \supseteq \gamma .
\end{aligned}
$$

Hence $x-y \in i_{V}(\tilde{g} ; \gamma)$. Note that $i_{F}(\tilde{f} ; \gamma)$ is a subfield of $F$ (see Theorem 5). Let $a \in i_{F}(\tilde{f} ; \gamma), x \in i_{V}(\tilde{g} ; \gamma)$, and $y \in a \circ x$. Then

$$
\widetilde{g}(y) \supseteq \bigcap_{z \in a \circ x} \widetilde{g}(z) \supseteq \widetilde{f}(a) \cap \widetilde{g}(x) \supseteq \gamma,
$$

and so $y \in i_{V}(\widetilde{g} ; \gamma)$ which shows that $a \circ x \subseteq i_{V}(\widetilde{g} ; \gamma)$. Therefore $i_{V}(\widetilde{g} ; \gamma)$ is a hypervector space over the field $i_{F}(\widetilde{f} ; \gamma)$ for all $\gamma \in \mathscr{P}(U)$.

Let $V$ and $W$ be hypervector spaces over $F$. A mapping $T$ : $V \rightarrow W$ is called linear transformation (see [3]) if it satisfies the following:

(i) $(\forall x, y \in V)(T(x+y)=T(x)+T(y))$,

(ii) $(\forall a \in F)(\forall x \in V)(T(a \circ x) \subseteq a \circ T(x))$.

Theorem 15. Let $V$ and $W$ be hypervector spaces over $F$ and let $(\tilde{f}, F)$ be an int-soft subfield of $F$. For any linear transformation $T: V \rightarrow W$, if $(\widetilde{g}, W)$ is an int-soft hypervector space of $W$ related to $(\tilde{f}, F)$, then $T^{-1}(\widetilde{g}, W)$ is an int-soft hypervector space of $V$ related to $(\tilde{f}, F)$.

Proof. Let $a, b \in F$ and $x, y \in V$. Since $T$ is a linear transformation, we have

$$
\begin{aligned}
& \bigcap_{z \in a \circ x+b \circ y} T^{-1}(\tilde{g})(z) \\
& =\bigcap_{z \in a \circ x+b \circ y} \tilde{g}(T(z))=\bigcap_{u \in a \circ x, v \in b \circ y} \tilde{g}(T(u+v)) \\
& \geq \bigcap_{\substack{T(u) \in a \circ T(x) \\
T(v) \in b \circ T(y)}} \tilde{g}(T(u+v)) \\
& \geq(\tilde{g}(T(x)) \cap \tilde{f}(a)) \bigcap(\tilde{g}(T(y)) \cap \tilde{f}(b)) \\
& =\left(T^{-1}(\tilde{g})(x) \cap \tilde{f}(a)\right) \bigcap\left(T^{-1}(\tilde{g})(y) \cap \tilde{f}(b)\right) .
\end{aligned}
$$

Obviously, $\widetilde{f}(1) \supseteq T^{-1}(\widetilde{g})(x)$ for all $x \in V$. It follows from Theorem 13 that $T^{-1}(\widetilde{g}, W)$ is a hypervector space of $V$ related to $(\tilde{f}, F)$.
Theorem 16. Let $V$ and $W$ be hypervector spaces over $F$ and let $(\tilde{f}, F)$ be an int-soft subfield of $F$. For any linear transformation $T: V \rightarrow W$, if $(\tilde{g}, V)$ is an int-soft hypervector space of $V$ related to $(\tilde{f}, F)$, then $T(\widetilde{g}, V)$ is an int-soft hypervector space of $W$ related to $(\tilde{f}, F)$.

Proof. Let $a, b \in F$ and $x, y \in W$. If at least one of $T^{-1}(x)$ and $T^{-1}(y)$ is empty, then the inclusion

$$
\begin{aligned}
& \bigcap_{z \in a \circ x+b \circ y} T(\tilde{g})(z) \\
& \quad \supseteq(\tilde{f}(a) \cap T(\widetilde{g})(x)) \bigcap(\tilde{f}(b) \cap T(\widetilde{g})(y))
\end{aligned}
$$

is clear. Assume that $T^{-1}(x)$ and $T^{-1}(y)$ are nonempty. Then there exists $u, v \in V$ such that $T(u)=x$ and $T(v)=y$. Thus

$$
\begin{aligned}
a \circ x+b \circ y & =a \circ T(u)+b \circ T(v) \\
& \supseteq T(a \circ u)+T(b \circ v)=T(a \circ u+b \circ v)
\end{aligned}
$$

since $T$ is linear. Hence $a \circ u+b \circ v \subseteq T^{-1}(a \circ x+b \circ y)$. Then

$$
\begin{aligned}
& \bigcap_{w \in a \circ x+b \circ y} T(\tilde{g})(w) \\
& =\bigcap_{\substack{w \in a \circ x+b o y \\
T(z)=w}} \tilde{g}(z) \\
& \supseteq \bigcap_{\substack{u \in T^{-1}(x), v \in T^{-1}(y) \\
z=z_{1}+z_{2}, z_{1} \in a o u, z_{2} \in b \circ v}}\left(\widetilde{g}\left(z_{1}\right) \cap \tilde{g}\left(z_{2}\right)\right) \\
& \supseteq(\tilde{f}(a) \cap \tilde{g}(u)) \bigcap(\tilde{f}(b) \cap \tilde{g}(v)) \\
& =(\tilde{f}(a) \cap T(\tilde{g})(x)) \bigcap(\tilde{f}(b) \cap T(\tilde{g})(y)) .
\end{aligned}
$$

Obviously, $\widetilde{f}(1) \supseteq T(\widetilde{g})(x)$ for all $x \in W$. Therefore $T(\widetilde{g}, V)$ is a hypervector space of $W$ related to $(\widetilde{f}, F)$ by Theorem 13 .

\section{Conflict of Interests}

The authors declare that there is no conflict of interests regarding the publication of this paper.

\section{Acknowledgment}

This work (RPP-2012-021) was supported by the fund of Research Promotion Program, Gyeongsang National University, 2012. The third author would like to thank Deanship of Scientific Research (DSR), King Abdulaziz University, Jeddah, for financial support.

\section{References}

[1] F. Marty, "Sur une generalization de la notion de groupe," in Proceedings of the 8th Congress des Mathematiciens Scandinaves, pp. 45-49, Stockholm, Sweden, 1934. 
[2] R. Ameri and O. Dehghan, "Fuzzy hypervector spaces based on fuzzy singletons," Computers \& Mathematics with Applications, vol. 61, no. 10, pp. 2933-2943, 2011.

[3] R. Ameri and O. R. Dehghan, "Fuzzy hypervector spaces," Advances in Fuzzy Systems, vol. 2008, Article ID 295649, 9 pages, 2008.

[4] L. A. Zadeh, "From circuit theory to system theory," Proceedings of the Institute of Radio Engineers, vol. 50, pp. 856-865, 1962.

[5] L. A. Zadeh, "Fuzzy sets," Information and Computation, vol. 8, pp. 338-353, 1965.

[6] L. A. Zadeh, "Toward a generalized theory of uncertainty (GTU) - an outline," Information Sciences, vol. 172, no. 1-2, pp. 1-40, 2005.

[7] D. Molodtsov, "Soft set theory-first results," Computers \& Mathematics with Applications, vol. 37, no. 4-5, pp. 19-31, 1999.

[8] P. K. Maji, A. R. Roy, and R. Biswas, "An application of soft sets in a decision making problem," Computers \& Mathematics with Applications, vol. 44, no. 8-9, pp. 1077-1083, 2002.

[9] P. K. Maji, R. Biswas, and A. R. Roy, "Soft set theory," Computers \& Mathematics with Applications, vol. 45, no. 4-5, pp. 555-562, 2003.

[10] D. Chen, E. C. C. Tsang, D. S. Yeung, and X. Wang, "The parameterization reduction of soft sets and its applications," Computers \& Mathematics with Applications, vol. 49, no. 5-6, pp. 757-763, 2005.

[11] N. Çağman, F. Çitak, and S. Enginoglu, "FP-soft set theory and its applications," Annals of Fuzzy Mathematics and Informatics, vol. 2, no. 2, pp. 219-226, 2011.

[12] F. Feng, "Soft rough sets applied to multicriteria group decision making," Annals of Fuzzy Mathematics and Informatics, vol. 2, no. 1, pp. 69-80, 2011.

[13] H. Aktaş and N. Çağman, "Soft sets and soft groups," Information Sciences, vol. 177, no. 13, pp. 2726-2735, 2007.

[14] U. Acar, F. Koyuncu, and B. Tanay, "Soft sets and soft rings," Computers \& Mathematics with Applications, vol. 59, no. 11, pp. 3458-3463, 2010.

[15] A. O. Atagün and A. Sezgin, "Soft substructures of rings, fields and modules," Computers \& Mathematics with Applications, vol. 61, no. 3, pp. 592-601, 2011.

[16] F. Feng, Y. B. Jun, and X. Zhao, "Soft semirings," Computers \& Mathematics with Applications, vol. 56, no. 10, pp. 2621-2628, 2008.

[17] Y. B. Jun, "Soft BCK/BCI-algebras," Computers \& Mathematics with Applications, vol. 56, no. 5, pp. 1408-1413, 2008.

[18] Y. B. Jun, H. S. Kim, and J. Neggers, "Pseudo $d$-algebras," Information Sciences, vol. 179, no. 11, pp. 1751-1759, 2009.

[19] Y. B. Jun, K. J. Lee, and A. Khan, "Soft ordered semigroups," Mathematical Logic Quarterly, vol. 56, no. 1, pp. 42-50, 2010.

[20] Y. B. Jun, K. J. Lee, and C. H. Park, "Soft set theory applied to ideals in $d$-algebras," Computers \& Mathematics with Applications, vol. 57, no. 3, pp. 367-378, 2009.

[21] Y. B. Jun, K. J. Lee, and J. Zhan, "Soft p-ideals of soft BCIalgebras," Computers \& Mathematics with Applications, vol. 58, no. 10, pp. 2060-2068, 2009.

[22] Y. B. Jun and C. H. Park, "Applications of soft sets in ideal theory of BCK/BCI-algebras," Information Sciences, vol. 178, no. 11, pp. 2466-2475, 2008.

[23] C. H. Park, Y. B. Jun, and M. A. Öztürk, "Soft WS-algebras," Korean Mathematical Society, vol. 23, no. 3, pp. 313-324, 2008.
[24] J. Zhan and Y. B. Jun, "Soft BL-algebras based on fuzzy sets," Computers \& Mathematics with Applications, vol. 59, no. 6, pp. 2037-2046, 2010.

[25] N. Çağman and S. Enginoğlu, "Soft set theory and uni-int decision making," European Journal of Operational Research, vol. 207, no. 2, pp. 848-855, 2010.

[26] M. S. Tallini, "Hypervector spaces," in Proceedings of the 4th International Congress on AHA, pp. 167-174, 1990. 


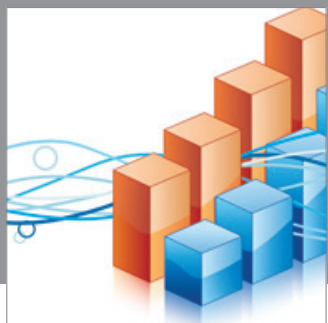

Advances in

Operations Research

mansans

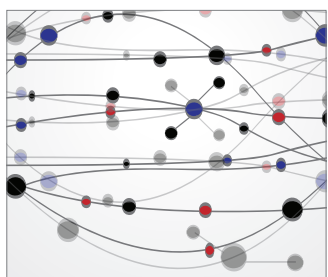

The Scientific World Journal
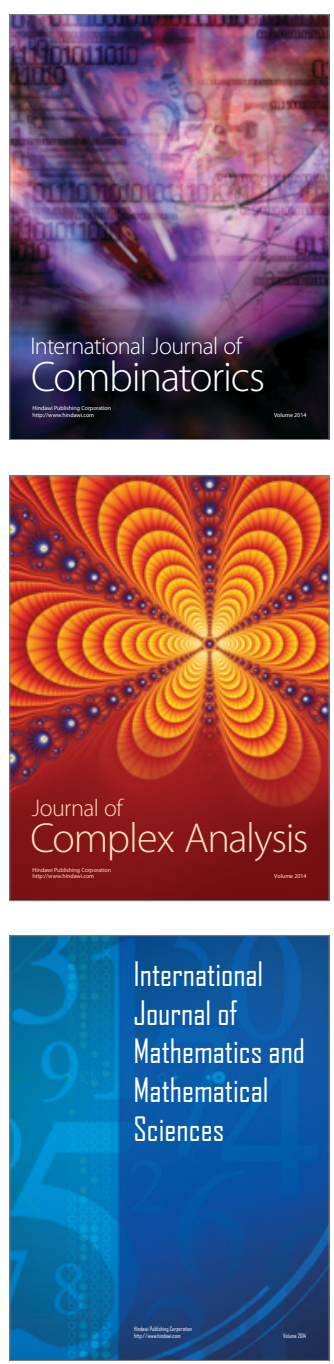
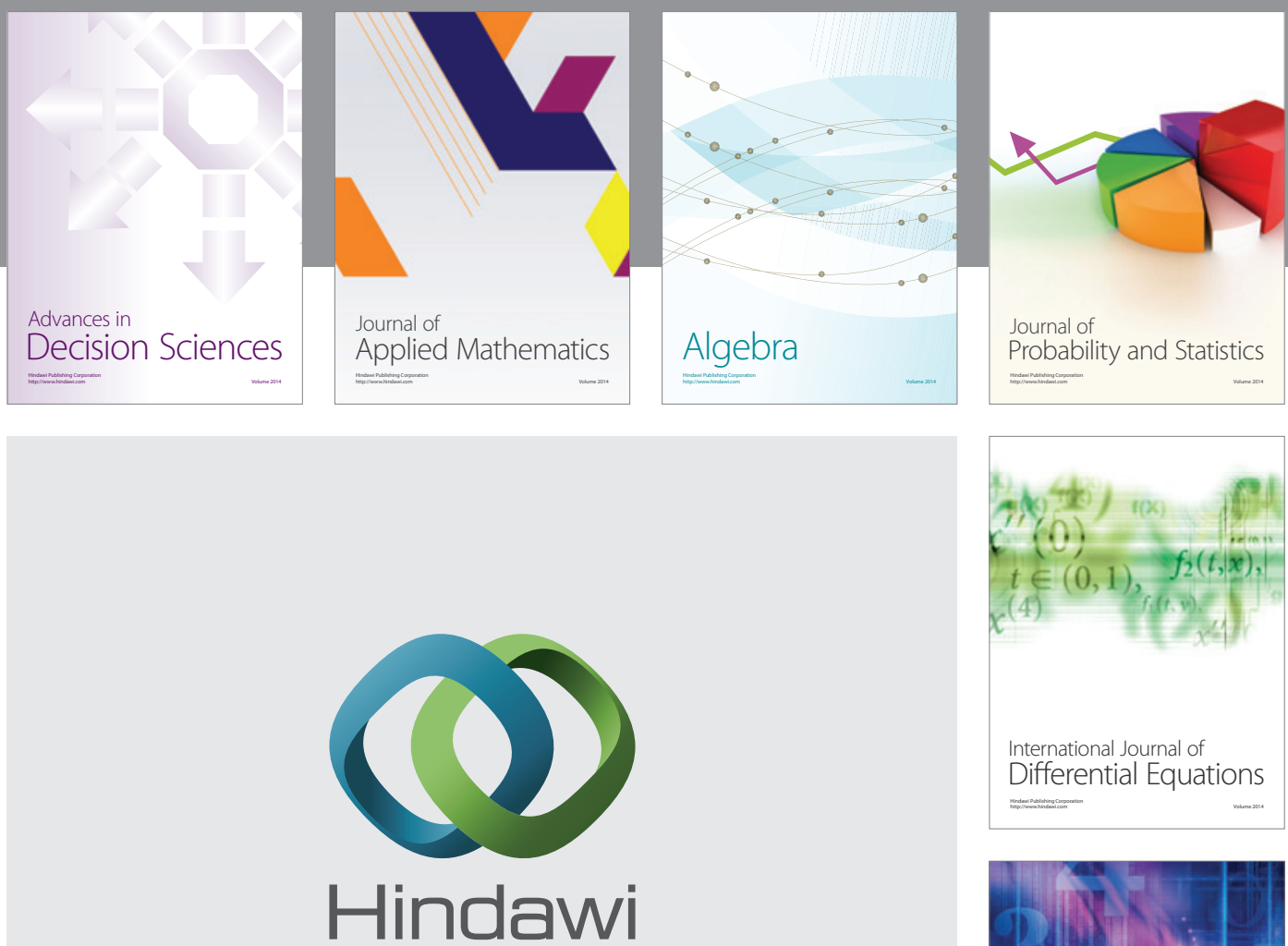

Submit your manuscripts at http://www.hindawi.com
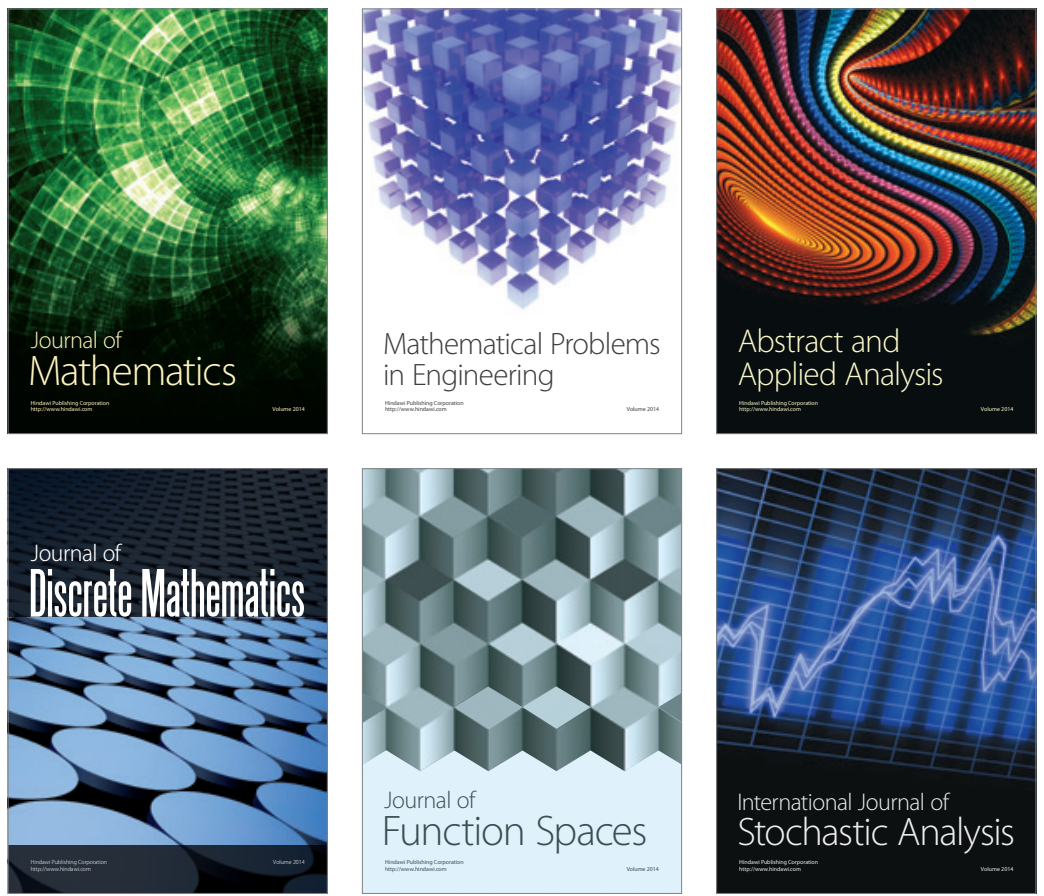

Journal of

Function Spaces

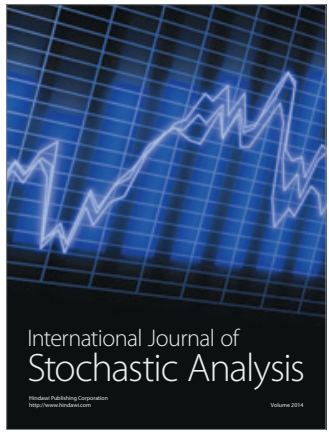

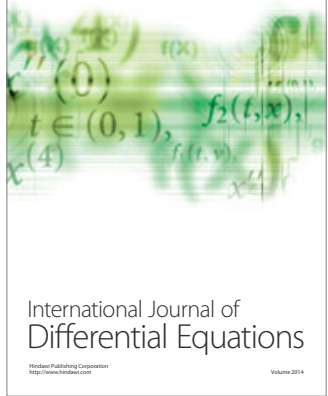
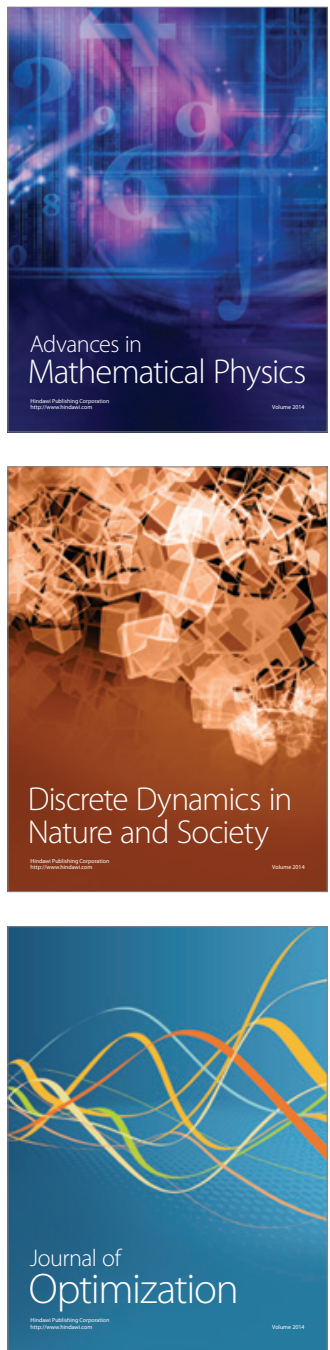